\title{
ON DERIVATIONS AND JORDAN DERIVATIONS THROUGH ZERO PRODUCTS
}

\author{
HOGER GHAHRAMANI
}

Abstract. Let $\mathscr{A}$ be a unital complex (Banach) algebra and $\mathscr{M}$ be a unital (Banach) $\mathscr{A}-$ bimodule. The main results describe (continuous) derivations or Jordan derivations $D: \mathscr{A} \rightarrow \mathscr{M}$ through the action on zero products, under certain conditions on $\mathscr{A}$ and $\mathscr{M}$. The proof is based on the consideration of a (continuous) bilinear map satisfying a related condition.

Mathematics subject classification (2010): 15A86, 47A07, 47B47, 47B49. algebra.

Keywords and phrases: Bilinear maps, derivation, Jordan derivation, zero (Jordan) product determined

\section{REFERENCES}

[1] J. Alaminos, M. Brešar, J. Extremera And A. R. Villena, Characterizing homomorphisms and derivations on $C^{*}$-algebras, Proc. R. Soc. Edinb. A 137 (2007), 1-7.

[2] J. Alaminos, M. Breš AR, J. Extremera And A. R. Villena, Maps preserving zero products, Studia Math. 193 (2009), 131-159.

[3] J. Alaminos, M. M. BrešAr, J. Extremera And A. R. Villena, Characterizing Jordan maps on $C^{*}$-algebras through zero products, Proc. Edinb. Math. Soc. 53 (2010), 543-555.

[4] M. BREŠAR, M. GRAŠić, J. S. ORTEGA, Zero product determined matrix algebras, Linear Algebra Appl. 430 (5/6) (2009), 1486-1498.

[5] M. BREŠAR, Multiplication algebra and maps determined by zero products, Linear and Multilinear Algebra, 60 (2012) 763-768.

[6] M. Burgos And J. S. ORTEGA, On mappings preserving zero products, Linear and Multilinear Algebra, 2012, in press.

[7] C. L. ChuAng, T. K. LeE, Derivations modulo elementary operators, J. Algebra 338 (2011), 56-70.

[8] H. GHAHRAMANi, Additive mappings derivable at nontrivial idempotents on Banach algebras, Linear and Multilinear Algebra, 60 (2012), 725-742.

[9] J. C. HoU AND X. L. ZHANG, Ring isomorphisms and linear or additive maps preserving zero products on nest algebras, Linear Algebra Appl. 387 (2004), 343-360.

[10] B. E. Johnson, Symmetric amenability and the nonexistence of Lie and Jordan derivations, Math. Proc. Cambd. Philos. Soc. 120 (1996), 455-473.

[11] C. Pearcy, D. Topping, Sum of small numbers of idempotent, Michigan Math. J. 14 (1967), 453465.

[12] N. K. Spanoudakis, Generalization of certain nest algebras results, Proc. Amer. Math. Soc. 115 (1992), 711-723. 